\title{
Video Tracking Based on Election Campaign Algorithm
}

\section{Qinghua Xie ${ }^{1, a^{*}}$, Xiangwei Zhang ${ }^{1, b}$, Wenge Lv $^{1, c}$ and Siyuan Cheng ${ }^{1, d}$}

${ }^{1}$ School of Electromechanical Engineering, Guangdong University of Technology, Guangzhou China

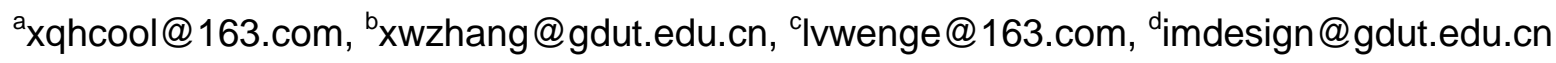

Keywords: Video Tracking; Grey Scale Feature; Focus Center; Election Campaign Algorithm

\begin{abstract}
Grey scale feature is not sensitive to the deformation and rotation in the image, and has good stability to detect similarity of image sequence. Using grey scale feature as object function, Election Campaign Algorithm considers the tracking windows as the effect ranges and focus center, the problem of video tracking converges to the optimal evaluation function to the central point. The focus center gets the feature different to the background and can be tracking the movement of the target. Random points around the focus center are generated to test the range of tracking window and determine the start point of focus center in the next image of the sequence. The simulation results show that the presented algorithm of video tracking is able to apply in real time, and the tracking performance is superior to conventional algorithms.
\end{abstract}

\section{Introduction}

Long time target tracking is a challenging task in the field of computer vision. In the process of target tracking, the tracking objects often disappear or occlusion, the object's angle or attitude change. The traditional tracking algorithm, but, does not have the target detection function, when the tracking target appeared again after the disappearance, it is easy to fail when the continuous movement of the target tracking.

The traditional tracking methods, such as optical flow method [1], are assumed to have no complete occlusion or target disappear. They implement the tracking frame by frame. The research works on this kind of methods are currently focused on to extend the action time to improve the tracking speed and precision or to develop a more reliable method [2]. These studies are not directly used in the long time target tracking problem.

The long time target tracking problem requires the tracking algorithm has its own detection ability, so that when the tracking target is occluded or disappear it will not lead to tracking failure. The target can be detected again when it reappear and then be tracking [3-6]. When the target disappeared in the camera view and appeared again, target appearance changes, detector training only by the initial target will lead to tracking error. Adaptive tracking method can solve the problem of target appearance changes in the tracking process. Such tracking methods can be roughly divided into two categories: the frame by frame update method [7-9] and the selection update method [8,9]. The frame by frame update method updates the target model with the best tracking results at the current time. In the case of high tracking accuracy, this method can solve the problem of the change of the target appearance. However, if the tracking accuracy is not high, this method may also accelerate the failure of tracking. The selection update method considering the tracking results are not always correct. It updates the model only if the model is close to the target or the new data of supervised learning are integrated into the model [10]. The above view is a summary of the TLD (tracking-earning-detection) algorithm [11]. TLD algorithm is the unknown object tracking, detection and tracking algorithm based on the long learning algorithm, the robustness of the algorithm framework is very strong, with a strong learning ability to recover, but with a similar target around, TLD has poor tracking performance [12-14].

In this paper, we use grey scale feature and position relation to detect the similarity of the tracking window and target model. The author uses the election campaign algorithm to find out the grey scale feature of random position and the position relation of them [15]. As an optimization process, the similarity of the tracking window should be detected and the target model update. When the tracking 
window and the target model are dissimilar, the model does not update. In the same time, the effect ranges of election campaign algorithm extend to detect more area around the focus center.

\section{Grey Scale Feature of Images}

Detecting of gray scale feature is not sensitive to the deformation and rotation of the target position in the image, and has good stability [16].

Set the image grey scale level is $L(O \sim l)$, the general $l$ is 255 . In order to speed up the computing and improve the real-time performance, the grey scale level is mapped to $m$ level, such as $m=8$, which is divided into 8 levels. So, the grey scale level of point is calculated by the Eq. 1 .

$G(r, c)=|x * m / l|$

Corresponding, assuming that the grey scale level of surrounding points at the center point $P(r, c)$, the objective function can be expressed as $S(r, c)$, the sum of levels of points. As is show in Eq. 2.

$$
S(r, c)=\underset{i=1,2, \cdots, n}{\operatorname{sum}}\left(\left|x_{i} * m / l\right|\right)
$$

So, the target localization problem in the process of comparing is transformed into the problem of the candidate target region which is the minimum value. When $P(r, c)=S(r, c)-I(r, c)$ is close to 0 , said the target and candidate regions are more similar; when $P(r, c)>R, R$ is the evaluation criterion and in this paper we set $R=\mathrm{n} / 2, \mathrm{n}$ is the number of comparing points, said the target and candidate region are dissimilar.

Fig. 1 shows the model of target and candidate regions, point $(1,2,3,4,6,7,8,9)$ is the points surrounding point 5 , all together the sum of 9 points gray scale level, such as Eq. 3, is used as the evaluation criterion of the center point.

$$
S(r, c)=\underset{i=1,2, \cdots, 9}{\operatorname{sum}}\left(\left|x_{i} * m / l\right|\right)
$$

After the criterion calculation of center points, the relation of center points is used as the fitness function of the optimization algorithm. Fig. 1 shows the relation model of center points. The numerical center of gravity of all the center points in target image can be found as the solid dot, hollow dots are center points. The relative position relation in target image is used when the same relation is detecting in the candidate image.

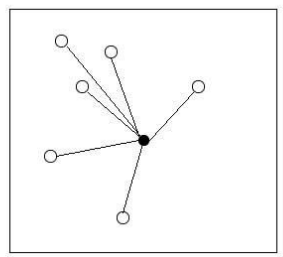

Figure 1. Comparing Model of Center Points

\section{Election Campaign Algorithm for Video Tracking}

Election campaign algorithm (ECA) is an optimization algorithm simulating election process [17]. Election candidates influence the voters round them, the voter's support is in proportion to the distance between the candidates and the voters. The voter will vote to the candidate which he pefer the most. The sum of location coordinates of every voters supported the candidate powered by its contribution is a new location coordinates, which is named support focus, it is the next position of the candidate. Such computational cycle is done continually until the function finds the position of the highest support, which is the global solution of the optimization problems.

Set the Algorithm Parameters. Main parameters of Election campaign algorithm are:

- The number of candidates.

- The number of voters.

- The number of floating voters.

- Target accuracy. 
Generate the Candidates and Calculate the Prestige of Them. Generated the define number of candidates in feasible solution field on the uniform distribution. Use the objective function to calculate the prestige of each candidate.

Calculate the Effect Range and Generate the Voters. If the target model update in the last image detection, the effect range does not change. When the stop condition is not reach, the range extends. The uniform distribution is employed to generate the voters in feasible solution field.

Compute the Investigate Mean Square of Candidates. Higher prestige of a candidate, smaller the mean square deviation of local voters, so that ECA is able to converge to local optimization solution rapidly and steadily.

Calculate the Supported of the Voters. The support of a voter is proportional to his prestige, and then the proportional constant will be reduced, so the prestige of a voter can used to denote the support of a voter directly. A voter may be influenced by several candidates; the voter should distribute his support to candidates proportionally on the magnitude of effect from candidate to voter.

Find the Support Focus of Candidates. A new position coordinate will achieve by means of summing the products of the support from the voters to the candidate and the position coordinate of the voters. It is named the support focus.

The support focus of a candidate is obtained by investigating, which depends on those voters whose distances to the candidate are nearer and the prestige is higher relatively. The next post of the candidate should be the support focus, where the candidate will have the higher support.

Calculate the Prestige of the Candidates and Compare the Prestige of the Voters with the Candidates. In order to jump out of local optimization solution and increase search rate, the prestige of candidates are compared to that of voters, if the prestige of a voter is higher than that of a candidate, the voter with higher prestige will substitute for the candidate and the that candidate of lower prestige will be eliminated in election.

Check Whether the Condition Is Reach. Check whether the condition is reach, otherwise return to step B to execute the period. Here, the condition could be the relative position relation in target image. Do that circularly until the highest support is not change.

$$
\left|\operatorname{sum}_{i=1,2, \cdots, 9} S_{c}\left(r_{i}^{r}, c_{i}^{r}\right)-\operatorname{sum}_{i=1,2, \cdots, 9} S_{t}\left(r_{i}, c_{i}\right)\right|<\xi
$$

$S_{c}$ represents the position relation of template, $S_{t}$ represents the position relation of target image region. Feature points of template $P_{r}\left(r_{i}, c_{i}\right)$ and points of target image region $P\left(r_{i}, c_{i}\right)$ have the same relative position relation. The right parameter on Eq. (5) is a very small number. Random rotation is set in the template as voters in ECA and the support focus in the previous ECA cycle is position to test more similar position. If the highest support is not change, the computations stop. When ECA stop, the candidate image is similar if Eq. 5 is workable.

\section{Experiment and Result}

Image set of Fig. 2 are few images in the test video 1 [18], the tracking window are draw a frame round in the images. Fig. 3 shows the tracking window and the focus center, target model are gray scale feature levels and the relation of random points in the effect range and the focus center, all the random points are generated on the normal distribution around the focus center.

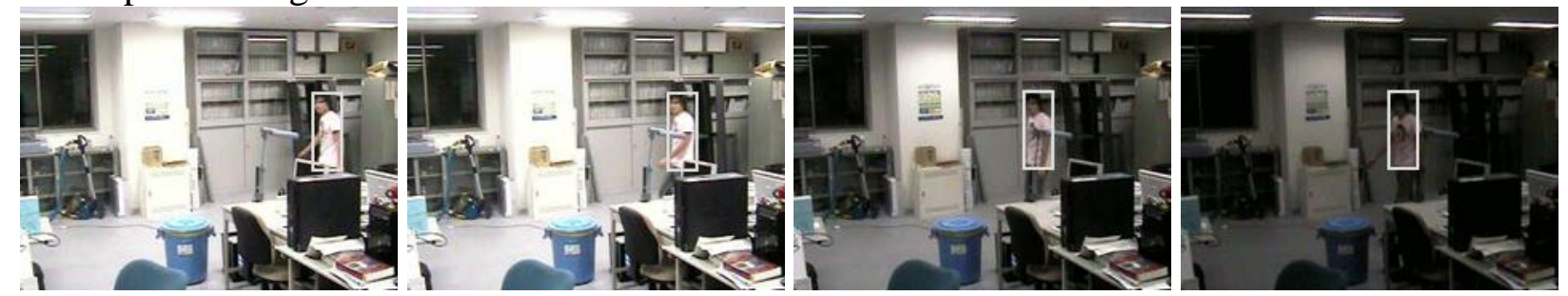

Figure 2. Tracking of test video 1 

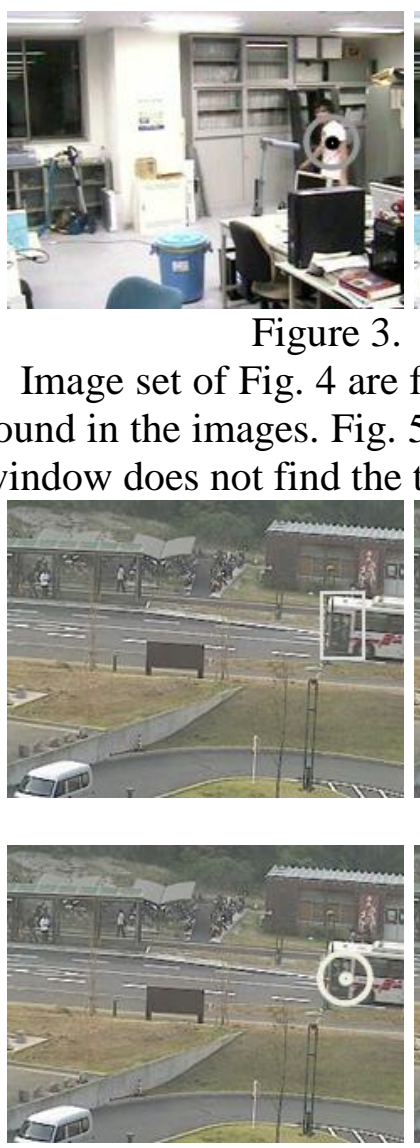

Figure 5. The tracking window and the target model of test video 2

Using Election campaign algorithm, the number of candidates define as 15 , the number of voters is 20 , the number of floating voters is 15 , and the target accuracy is set as 2.5 . The effect range is set as the radius of the tracking window, every tracking failure happens, the range extend to be $110 \%$ of the last range when target model is not update.

\section{Conclusions}

With the method of comparing the relation of gray scale feature levels, the similar area of target model can be detected. It is easier achievable and less computation then TLD algorithm, but has the similar principle. Election campaign algorithms for video tracking detect the similar image area similar to the target model, and in the same time update the model with selection. This is a potential solvent for long time target tracking. For future work, object function of the optimization process will be test in the detecting of the tracking window.

\section{References}

[1] Lucas B. D., Kanade T., An iterative image registration technique with an application to stereo vision, Proceedings of International Joint Conference on Artificial Intelligence, Menlo Park, California, AAAI Press, 1981, pp. 674-679.

[2] Comaniciu D., Ramesh V., Meer P. Kernel-based objects tracking, IEEE Transactions on Pattern Analysis and Machine Intelligence, 2003, 25(5), pp. 564-577.

[3] Lepetit V., Lagger P., Fua P., Randomized trees for real-time key-point recognition, Proceedings of IEEE Conference on Computer Vision and Pattern Recognition, New York, IEEE Press, 2005, pp. 775-781.

[4] Andriluka M., Roth S., Schiele B., People-tracking-by-detection and people-detection-by-tracking, Proceedings of IEEE Conference on Computer Vision and Pattern Recognition, New York, IEEE Press, 2008,pp. 1-8. 
[5] Viola P., Jones M., Rapid object detection using a boosted cascade of simple features, Proceedings of IEEE Conference on Computer Vision and Pattern Recognition, New York, IEEE Press, 2001, pp. 511-518.

[6] Ozuysal M., Lepetit V., Fleuret F., et al., Feature harvesting for tracking-by-detection, Proceedings of European Conference on Computer Vision, Berlin, Germany, Springer-Verlag, 2006, pp. 592-605.

[7] Collins R. T., Liu Y., Leordeanu M., Online selection of discriminative tracking features, IEEE Transactions on Pattern Ana-lysis and Machine Intelligence, 2005, 27(10), pp. 1631-1643.

[8] Grabner H.,Bischof H., On-line boosting and vision, Proceedings of IEEE Conference on Computer Vision and Pattern Recognition, New York, IEEE Press, 2006, pp. 260-267.

[9] Avidan S., Ensemble tracking, IEEE Transactions on Pattern Analysis and Machine Intelligence, 2007, 29 (2), pp. 261-271.

[10] Matthews L., Ishikawa T., Baker S., The template update problem, IEEE Transactions on Pattern Analysis and Machine Intelligence, 2004, 26( 6), pp. 810-815.

[11] Grabner H., Leistner C., Bischof H., Semi-supervised on-line is boosting for robust tracking, Proceedings of European Conference on Computer Vision, Berlin, Germany, Springer-Verlag, 2008, pp. 234-247.

[12] KALAL Z., MIKOLAJCZYK K., MATAS J., Face TLD: Tracking-learning-detection applied to faces, Proc. ICIP 2010. Hong Kong: IEEE Press, 2010, pp. 3789-3792.

[13] VIOLA P., PLATT J., ZHANG C., Multiple instance Boosting for object detection, Advances in Neural Information Processing Systems, 2006(18), pp. 1417-1424.

[14]CALONDER M., LEPETIT V., FUA P., Fast key point recognition using random ferns, IEEE Trans, Pattern Analysis and Machine Intelligence, 2010, 32(3), pp. 448-461.

[15] Wenge Lv, Qinghua Xie, Zhiyong Liu, Deyuan Li, et al., Verifying Election Campaign Optimization Algorithm by Several Benchmarking Functions, Lecture Notes in Computer Science, 6146 LNCS, 2, pp. 582-57.

[16]Fang B., Leung C.H., Tang Y.Y., et al., Offline signature verification with generated training samples, IEEE Proc.-Vis. Images Signal Process, 2002, 149(2).

[17] Wenge Lv, Chunhua He, DeyuanLi, et al., Election Campaign Optimization Algorithm. Procedia Computer Science, 1, pp. 1371-1380.

[18] Camera Parameter Changes in Indoor Scene, Department of Advanced Information Technology, Kyushu University, http://limu.ait.kyushu-u.ac.jp/dataset/en/index.html

[19]Bus Stop in the Evening, Department of Advanced Information Technology, Kyushu University, http://limu.ait.kyushu-u.ac.jp/dataset/en/index.html 